\title{
EXPRESSION OF ACIDIC FIBROBLAST GROWTH FACTOR IN BARRETT'S ESOPHAGUS AND ASSOCIATED ESOPHAGEAL ADENOCARCINOMA
}

Robert A. Soslow, MD

Liang Ying, BS

Nasser K. Altorki, MD
Objective: Adenocarcinoma of the esophagus is generally attributed to the neoplastic transformation of intestinal metaplastic lesions (Barrett's esophagus). On the basis of our preliminary data that showed significant acidic fibroblast growth factor mRNA and protein expression in adenocarcinoma of the esophagus, we studied expression of fibroblast growth factor in esophageal adenocarcinoma and its precursor lesions, intestinal metaplasia, low-grade dysplasia, and high-grade dysplasia. Fibroblast growth factor belongs to a family of polypeptides that are involved in differentiation and cellular proliferation. Methods: We examined 30 esophagectomy specimens that were resected for adenocarcinoma $(n=27)$ and high-grade dysplasia $(n=3)$. After confirmation of the diagnosis by routine light microscopy, the index lesions (invasive carcinomas) and adjoining Barrett's mucosa were evaluated with a monoclonal antibody against human acidic fibroblast growth factor. The results are expressed with the use of an immunoreactive score that allows distinction between weak, moderate, and strong immunoreactivity. Results: Adenocarcinoma demonstrated a moderate-to-strong mean immunoreactive score of 8 . In contrast, high-grade dysplasia demonstrated a weak-to-moderate mean score of 4.5 , which was significantly different $(p<0.05)$. Intestinal metaplasia and low-grade dysplasia displayed even weaker expression of fibroblast growth factor, with a negligible immunoreactive score less than $1(p<0.005)$. Seventy-five percent of evaluable cases demonstrated an increasing degree of fibroblast growth factor expression in the spectrum of lesions ranging from metaplasia to dysplasia and carcinoma. Conclusions: These data indicate that in patients with adenocarcinoma arising in association with Barrett's esophagus, fibroblast growth factor is generally sequentially accumulated in the progression from metaplasia to neoplasia. This progression may affect future investigation into the role of fibroblast growth factors in tumorigenesis and, possibly, the application of fibroblast growth factor immunohistochemistry to diagnosis. (J Thorac Cardiovasc Surg 1997;114:838-43)
From the Departments of Pathology and Cardiothoracic Surgery, New York Hospital-Cornell Medical Center, New York, N.Y.

Read at the Seventy-seventh Annual Meeting of The American Association for Thoracic Surgery, Washington, D.C., May 4-7, 1997.

Received for publication May 7, 1997; revisions requested June 2, 1997; revisions received June 18, 1997; accepted for publication June 19, 1997.

Address for reprints: Nasser K. Altorki, MD, New York Hospital-Cornell Medical Center, 525 East 68th St., Division of Cardiothoracic Surgery, New York, NY 10021.

Copyright (C) 1997 by Mosby-Year Book, Inc.

$0022-5223 / 97 \$ 5.00+0 \quad \mathbf{1 2 / 6 / 8 4 2 7 7}$
$B$ arrett's esophagus poses numerous diagnostic and therapeutic difficulties. Of compelling interest is the association of intestinal metaplasia and superimposed dysplasia with esophageal adenocarcinoma. These putative precursors of carcinoma may place a patient at risk for the development of invasive adenocarcinoma. They also provoke research interest because of the opportunity they provide to examine sequential changes in oncogenes, tumor suppressor genes, and cell cycle regulators, as well as adhesion molecules and angiogenesis factor expression that are associated with the development of invasive carcinoma. In this study we 
Table I. Staining

\begin{tabular}{|c|c|}
\hline Score & Description \\
\hline & Staining intensity \\
\hline $1+$ & $\begin{array}{l}\text { Weak, uniform, brown, cytoplasmic coloration just } \\
\text { beyond the level of any background staining }\end{array}$ \\
\hline $2+$ & Moderate, uniform, brown, cytoplasmic coloration \\
\hline $3+$ & Strong, uniform, brown, cytoplasmic staining \\
\hline \multirow[t]{2}{*}{$4+$} & The darkest uniform, brown, cytoplasmic staining \\
\hline & Staining distribution \\
\hline 1 & $1 \%-25 \%$ reactive cells \\
\hline 2 & $26 \%-50 \%$ reactive cells \\
\hline 3 & $51 \%-75 \%$ reactive cells \\
\hline \multirow[t]{2}{*}{4} & $76 \%-100 \%$ reactive cells \\
\hline & Immunoreactive scores $*$ \\
\hline $1-3$ & Weak expression \\
\hline $4-8$ & Moderate expression \\
\hline $9-12$ & Strong expression \\
\hline $13-16$ & Very strong expression \\
\hline
\end{tabular}

${ }^{*}$ Computed by multiplying staining intensity by staining distribution.

present data concerning acidic fibroblast growth factor (FGF-1), which belongs to a family of polypeptides involved in differentiation and cellular proliferation. ${ }^{1}$

Although the data linking FGF-1 to malignant neoplasms are not extensive, some of its reported biologic functions are potentially related to tumor progression and invasion. FGFs are mitogenic, promote angiogenesis, and play an important role in tissue repair, including fibrosis. ${ }^{2,3}$ FGF-1 binding to its high-affinity cytoplasmic membrane receptor promotes cellular proliferation. ${ }^{2}$ Therefore FGF-1 expression may be indicative of autocrine growth in tumors that express such receptors. ${ }^{4}$ Angiogenesis has been shown to be important in the maintenance of invasive tumor growth and has been correlated with prognosis in numerous organ systems. ${ }^{5-16}$ It is conceivable that the function of FGF-1 in tissue repair may also apply to the interaction between invasive tumors and the extracellular matrix. FGF-1 expression has been documented in transitional cell carcinoma of the urinary bladder ${ }^{17}$ pancreatic ductal adenocarcinoma, ${ }^{18}$ central nervous system gliomas, ${ }^{19}$ thyroid carcinomas, ${ }^{20}$ head and neck tumors, ${ }^{21}$ renal adenocarcinomas, ${ }^{19}$ and in chronic pancreatitis. ${ }^{22}$ It has been suggested that FGF-1 expression is correlated with advanced tumor stage and may adversely affect survival. ${ }^{17,18}$

We have previously shown that FGF mRNA and protein are both highly expressed in esophageal adenocarcinoma. ${ }^{23}$ In the present study we evaluated the hypothesis that FGF-1 expression is ac-
Table II. Summary of FGF-1 reactivity

\begin{tabular}{lcccc}
\hline & $\begin{array}{c}\text { Positive } \\
\text { No. }\end{array}$ & $\begin{array}{c}\text { Negative } \\
\text { No. }\end{array}$ & $\begin{array}{c}\text { Percentage of } \\
\text { positive cases }\end{array}$ & Average IRS \\
\hline IM & 6 & 11 & 35 & $<1$ \\
LGD & 3 & 10 & 23 & $<1$ \\
HGD & 12 & 6 & 67 & 4.5 \\
CA & 24 & 3 & 89 & 8.0 \\
\hline
\end{tabular}

$I M$, Intestinal metaplasia; LGD, low-grade dysplasia; $H G D$, high-grade dysplasia: $C A$, infiltrating adenocarcinoma.

quired sequentially in the metaplasia-dysplasia-carcinoma sequence.

\section{Materials and methods}

Case selection and slide review. We reviewed 27 randomly selected esophagectomy specimens that contained invasive adenocarcinoma and three additional esophagectomy specimens that contained high-grade dysplasia occurring in the setting of intestinal (Barrett's) metaplasia. The reported diagnoses were confirmed on review of sections stained with hematoxylin and eosin. The criteria of Lewin and Appelman ${ }^{24}$ to distinguish the following entities were used: gastric metaplasia, intestinal metaplasia, dysplasia, and infiltrating adenocarcinoma.

Immunohistochemistry. Formalin-fixed paraffin-embedded sections were selected for immunoperoxidase staining on the basis of the presence of invasive adenocarcinoma and, if present, intestinal metaplasia, low-grade dysplasia, and high-grade dysplasia. The tissue was incubated with a monoclonal antibody against human FGF-1 (Upstate Biotechnology Inc., Lake Placid, N.Y.) at an antibody dilution of 1:100. We used a biotinylated antimouse immunoglobulin IgG secondary antibody and streptavidin-peroxidase complex. Diaminobenzidine tetrahydrochloride was used as the substrate. Full-thickness sections of stomach wall, taken from the gastric fundus, were used as positive and negative control specimens. Negative control specimens were incubated with horse serum instead of with the primary antibody.

Interpretation. On the basis of the pattern and intensity of reactive cells in control tissue, we assigned an intensity score $(0$ to $4+)$ and a distribution score (estimated percentage of reactive cells) to describe staining of study cases. The criteria for scoring staining intensity follow: $1+$, uniform brown cytoplasmic coloration that is just beyond the level of any background staining, if present; $2+$, moderate, uniform brown cytoplasmic coloration; $3+$, strong, uniform brown cytoplasmic coloration; $4+$, the darkest uniform brown cytoplasmic coloration identified. An immunoreactive score (IRS), modified from the German IRS, ${ }^{25}$ was computed to account for both the distribution and intensity of staining and the classification of staining as weak, moderate, strong, or very strong. To calculate the IRS, we assigned the following points for staining distribution: $1,1 \%$ to $25 \% ; 2,26 \%$ to $50 \% ; 3,51 \%$ to $75 \% ; 4,76 \%$ to $100 \%$. These points were then multiplied by the staining intensity score, for a range of potential IRSs from 0 to 16 . Weak staining was defined as an IRS that ranged from 1 to 3 ; moderate 

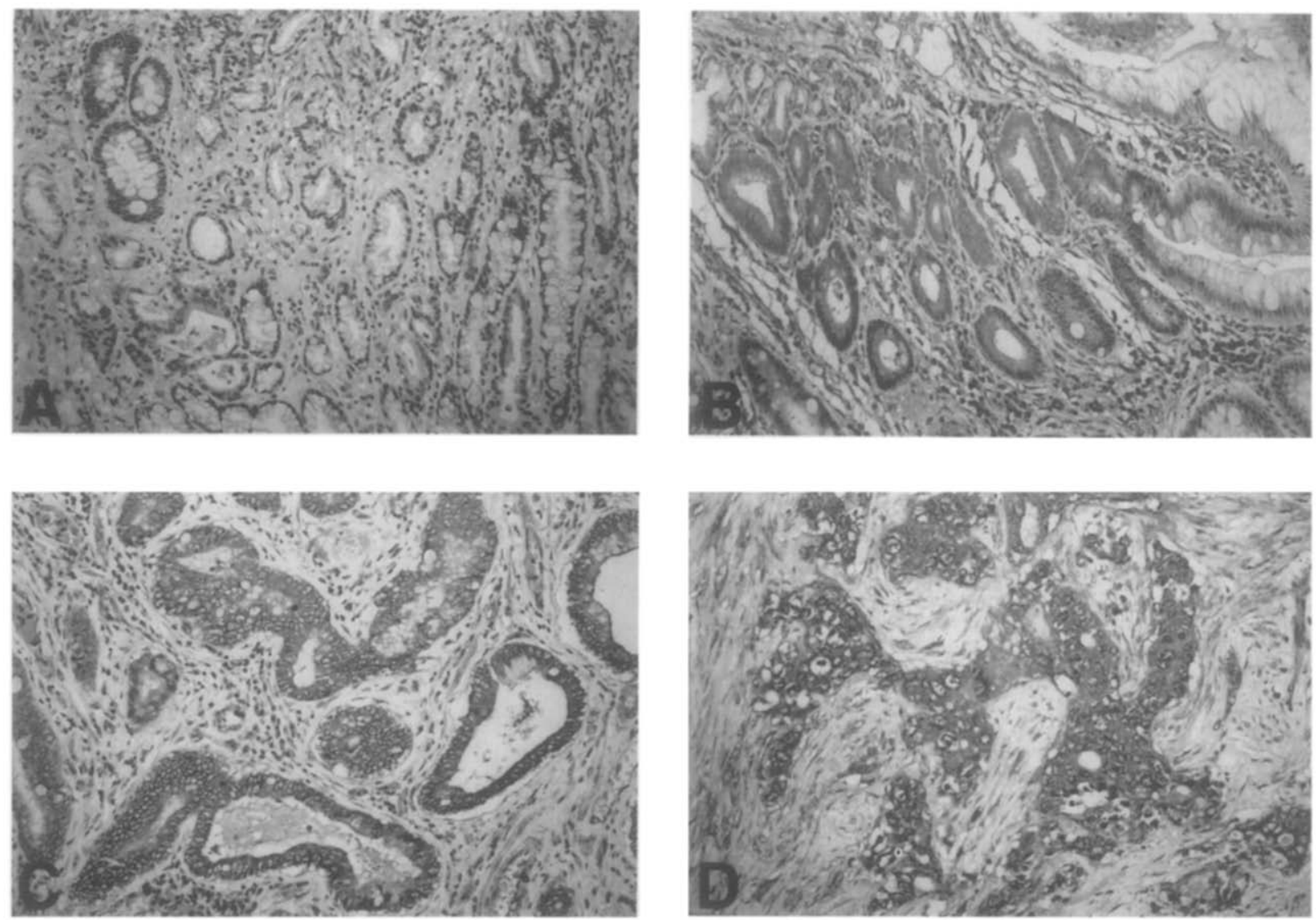

Fig. 1. FGF-1 expression in Barrett's esophagus and esophageal adenocarcinoma. A, Intestinal metaplasia: Metaplastic mucosa, which includes intestinal-type goblet cells, does not express FGF-1. B, Low-grade dysplasia: A subpopulation of dysplastic glands demonstrate very weak (light gray) cytoplasmic coloration with the FGF-1 monoclonal antibody. C, High-grade dysplasia: All the dysplastic glands demonstrate moderate-to-strong (dark gray) cytoplasmic coloration with the FGF-1 monoclonal antibody. D, Adenocarcinoma: This jagged, infiltrating nest of poorly differentiated adenocarcinoma strongly expresses FGF-1. Note the dark-gray cytoplasmic coloration.

staining was 4 to 8 , strong staining was 9 to 12 , and very strong staining was 13 to 16 (Table I).

\section{Results}

Histology. Twenty-seven esophagectomy specimens were found to contain infiltrating adenocarcinoma and three specimens demonstrated highgrade dysplasia, occurring in the background of intestinal metaplasia. Twenty-two of the infiltrating carcinomas contained intestinal metaplastic or dysplastic epithelium (or both) in the esophagectomy specimen.

\section{Immunohistochemistry}

Controls. Seven of 17 control gastric fundic mucosae demonstrated diffuse, 1 to $2+$ intensity staining of parietal cell cytoplasm (IRS $=4$ to 8 ). The foveolar (surface) epithelial cells were uniformly negative with the anti-FGF-1 antibody (IRS $=0$ ). When squamous mucosa was present for immunohistochemical evaluation, we identified diffuse, $1+$ diffuse cytoplasmic staining (IRS $=1$ to 4 ). Rare examples demonstrated scattered, weakly reactive nuclei. Ten cases did not demonstrate significant staining of control gastric fundic mucosa. The muscularis propria and endothelium showed diffuse, 1 to $2+$ cytoplasmic staining (IRS $=4$ to 8 ).

Adenocarcinoma. IRSs ranged from 0 to 12 (mean IRS $=8.0$, moderate staining bordering on strong staining). Twenty-four of 27 infiltrating adenocarcinomas reviewed contained significant numbers of tumor cells reactive with the anti-FGF-1 antibody. On average, FGF-1 reactive carcinomas 
were moderately to strongly reactive (mean IRS $=$ 8.5). All but one reactive case demonstrated moderate or strong FGF-1 reactivity; 15 of the adenocarcinomas studied $(56 \%)$ were strongly reactive with anti-FGF-1, eight were moderately reactive, and one was weakly reactive (Table II, Fig. 1). Many of the tumors demonstrated a range of staining intensity; the average staining intensity was considered when calculating the IRS.

High-grade dysplasia. Twelve of 18 high-grade dysplasias contained cells that were marked with anti-FGF-1. IRSs ranged from 0 to 12 (mean IRS = 4.5 , moderate staining bordering on weak staining). Most reactive cases were moderately or strongly positive for FGF-1 (8/12). The remaining four cases were only weakly reactive. The mean high-grade dysplasia IRS of 4.5 is significantly different from the mean infiltrating adenocarcinoma IRS of $8(p<$ 0.05 ) (two-tailed $t$ test) (Table II, Fig. 1).

Low-grade dysplasia and intestinal metaplasia. Only rare, weak staining was identified in cases of low-grade dysplasia; three of 13 samples of lowgrade dysplasia were positive for FGF-1, for an overall mean IRS of less than 1 . Only six of 17 metaplasias without dysplasia expressed any FGF-1 at all, for an overall mean IRS of less than 1 . The mean intestinal metaplasia and low-grade dysplasia IRSs of less than 1 are significantly different from the mean high-grade dysplasia IRS of 4.5 ( $p<$ 0.005 ) (two-tailed $t$ test) (Table II, Fig. 1).

Metaplasia-dysplasia-carcinoma sequence. Sixteen specimens contained at least three of the following lesions: intestinal metaplasia, low-grade dysplasia, high-grade dysplasia, or infiltrating adenocarcinoma. These cases were considered evaluable for FGF-1 expression trends in the metaplasia-dysplasia-carcinoma sequence. Ten of 16 cases demonstrated a definite sequential increase in FGF-1 immunoreactivity from intestinal metaplasia to dysplasia and to carcinoma. Two additional cases showed a probable trend in increasing FGF-1 reactivity. Together, these 12 cases account for $75 \%$ of evaluable esophagectomy specimens. Two further cases failed to exhibit sequential increases in FGF-1 staining. Finally, two cases were negative for FGF-1 (Table III).

\section{Discussion}

Our data indicate that esophageal adenocarcinomas are largely FGF-1 reactive and that FGF-1 expression correlates with the histologic grade of esophageal adenocarcinoma precursor lesions.
Table III. FGF-I immunoreactive scores in esophagectomy specimens evaluable for the metaplasia-dysplasia-carcinoma sequence

\begin{tabular}{ccccc}
\hline & \multicolumn{4}{c}{ Increasing $F G F-1$ expression in the MDCS } \\
\cline { 2 - 5 } Case & $I M$ & $L G D$ & $H G D$ & $C A$ \\
\hline 4 & 0 & 0 & 3 & \\
17 & 0 & 2 & 9 & 12 \\
18 & 0 & 0 & 0 & 4 \\
21 & 0 & 0 & 0 & 4 \\
22 & 1 & 1 & & 12 \\
23 & 0 & 2 & 3 & 12 \\
26 & 1 & & 6 & 9 \\
27 & 0 & 0 & 2 & \\
28 & 0 & 0 & 0 & 12 \\
29 & & & & \\
& & & 0 & 12 \\
5 & 1 & & 12 & 10 \\
6 & 0 & & & \\
& & & & \\
8 & & & & \\
24 & 1 & & & \\
& & & & \\
\hline
\end{tabular}

$M D C S$, Metaplasia-dysplasia-carcinoma sequence; $I M$, intestinal metaplasia; $L G D$, low-grade dysplasia; $H G D$, high-grade dysplasia; $C A$, infiltrating adenocarcinoma.

Moderate to strong FGF-1 expression occurs in almost all esophageal adenocarcinomas. This contrasts with the weak to moderate FGF-1 expression in high-grade dysplasia and the almost negligible FGF-1 expression in low-grade dysplasia and intestinal metaplasia. Furthermore, we show a trend toward increasing FGF-1 immunoreactivity within individual cases that contain lesions evaluable for the metaplasia-dysplasia-carcinoma sequence. Subjective interpretation of immunohistochemistry was minimized with the use of the German IRS, which has been shown to be equivalent to an image analysis-based quantitation of immunoreactivity. ${ }^{25}$

The trend toward increasing FGF-1 expression with the increasing histologic severity of Barrett's metaplasia and dysplasia allows us to make comparisons with the progressive accumulation of tumor suppressor gene and oncogene abnormalities in similar lesions. A greater degree of p53 overexpression, S-phase and DNA ploidy abnormalities, as well as p53 mutations, have been reported in high-grade dysplasias and adenocarcinomas, as compared with intestinal metaplasias and low-grade dysplasias. ${ }^{26-30}$ These data, in addition to ours, support the theory that high-grade dysplasia is an adenocarcinoma precursor lesion. Although some studies have described similar abnormalities in intestinal metaplasia and low-grade dysplasia, there is less molecular and 
genetic data that links these lesions closely with adenocarcinoma. ${ }^{26-28}$ Only three of our esophagectomy specimens contained both low-grade dysplasia and infiltrating adenocarcinoma without coexistent high-grade dysplasia, and all such cases demonstrated negligible FGF-1 expression in the low-grade dysplasia. Further investigation is needed to draw definite conclusions from these observations.

The link between stromal reactions and FGF-1 is interesting given the characteristic desmoplastic response of stroma infiltrated by adenocarcinoma. However, we found rare cases in which nonneoplastic epithelium, including intestinal metaplastic epithelium, expressed FGF-1 in an eroded region or adjacent to an ulcer. We think that FGF-1 may be expressed by nonneoplastic epithelium, especially in sites of tissue injury; this has been reported in cases of chronic pancreatitis. ${ }^{22}$

Although FGF-1 expression is certainly conducive to tumor progression, it is probably not necessary, given our inability to demonstrate FGF-1 expression in three of the adenocarcinomas studied. Other investigations of FGF-1 expression have shown that the absence of FGF-1 mRNA correlates with failure to detect FGF-1 by immunohistochemical methods, ${ }^{22}$ suggesting that FGF-1-negative cases in this study do not likely express FGF-1 mRNA. We have excluded technical reasons for this negative FGF-1 immunostaining because of appropriate FGF-1 expression in the positive internal and external control specimens. Altered or mutated FGF-1, not recognized by the monoclonal antibody used, may have given this result. Possibly, other fibroblast growth factors, such as FGF-2, contributed to tumor progression. The FGF-1-negative adenocarcinoma of case 8 was associated with high-grade dysplasia that showed moderate FGF-1 reactivity. In rare cases, FGF-1 expression may be altered in the progression from high-grade dysplasia to infiltrating adenocarcinoma; we do not think these data indicate that high-grade dysplasia and infiltrating adenocarcinoma arise from different clones.

In addition to its theoretic role in tumor progression, FGF-1 immunohistochemistry may be useful in the diagnosis of Barrett's esophagus and esophageal adenocarcinoma. Because of its preferential expression in high-grade dysplasia and infiltrating adenocarcinoma, FGF-1 immunoreactivity may become useful as a means to distinguish low-grade from high-grade dysplasia, which is of clinical relevance. However, our observation that low-grade lesions may express FGF-1 in ulcerated areas may compli- cate routine use. A prospective investigation to determine the biologic potential of FGF-1-reactive dysplasias would be informative.

\section{REFERENCES}

1. Goldfarb M. The fibroblast growth factor family. Cell Growth Differ 1990;1:439-45.

2. Klagsbrun M. The fibroblast growth factor family: structural and biological properties. Prog Growth Factor Res 1989;1: 207-35.

3. Givol D, Yayon A. Complexity of FGF receptors: genetic basis for structural diversity and functional specificity. FASEB J 1992;6:3362-9.

4. Jaye M, Lyall RM, Mudd R, Schlessinger J, Srver N. The expression of acidic fibroblast growth factor cDNA confers growth advantage and tumorigenesis to Swiss $3 \mathrm{~T} 3$ cells. EMBO J 1988;7:963-9.

5. Obermair A, Kohlberger P, Bancher-Todesca D, Tempfer C, Sliutz G, Leodolter S, et al. Influence of microvessel density and vascular permeability factor/vascular endothelial growth factor expression on prognosis in vulvar carcer. Gynecol Oncol 1996;63:204-9.

6. Gleich LL, Biddinger PW, Pavelic ZP, Gluckman JL. Tumor angiogenesis in $\mathrm{T} 1$ oral cavity squamous cell carcinoma: role in predicting tumor aggressiveness. Head Neck 1996;18: 343-6.

7. Fontanini G, Vignati S, Pacini F, Pollina L, Basolo F. Microvessel count: an indicator of poor outcome in medullary thyroid carcinoma but not in other types of thyroid carcinoma. Mod Pathol 1996;9:636-41.

8. Takebayashi Y, Aklyama S, Yamada K, Akiba S, Aikou T. Angiogenesis as an unfavorable prognostic factor in human colorectal carcinoma. Cancer 1996;78:226-31.

9. Harpole DH Jr, Richards WG, Herndon JE II, Sugarbaker DJ. Angiogenesis and molecular biologic substaging in patients with stage I non-small cell lung cancer. Ann Thorac Surg 1996;61:1470-6.

10. Yoshino S, Kato M, Okada K. Prognostic significance of microvessel count in low stage renal cell carcinoma. Int J Urol 1995;2:156-60.

11. Bochner BH, Cote RJ, Weidner N, Groshen S, Chen SC, Skinner DG, et al. Angiogenesis in bladder cancer: relationship between microvessel density and tumor prognosis. J Nat1 Cancer Inst 1995;87:1603-12.

12. Zatterstrom UK, Brun E, Willen R, Kjellen E, Wennerberg J. Tumor angiogenesis and prognosis in squamous cell carcinoma of the head and neck. Head Neck 1995;17:312-8.

13. Hollingsworth HC, Kohn EC, Steinberg SM, Rothenberg ML, Merino MJ. Tumor angiogenesis in advanced stage ovarian carcinoma. Am J Pathol 1995;147:33-41.

14. Maeda K, Chung YS, Takatsuka S, Ogawa Y, Sawada T, Yamashita $\mathrm{Y}$, et al. Tumor angiogenesis as a predictor of recurrence in gastric carcinoma. J Clin Oncol 1995;13:477 81.

15. Vartanian RK, Weidner N. Correlation of intratumoral endothelial cell proliferation with microvessel density (tumor angiogenesis) and tumor cell proliferation in breast carcinoma. Am J Pathol 1994;144:1188-94.

16. Dickinson AJ, Fox SB, Persad RA, Hollyer J, Sibley GN. Quantification of angiogenesis as an independent predictor 
of prognosis in invasive bladder carcinomas. $\mathrm{Br} \mathrm{J}$ Urol 1994;74:762-6.

17. Yoshimura K, Eto H, Miyake H, Hara I, Arakawa S, Kamidono S. Messenger ribonucleic acids for fibroblast growth factors and their receptor in bladder and renal cell carcinoma cell lines. Cancer Lett 1996;103:91-7.

18. Yamanaka Y, Friess H, Buchler M, Beger HG, Uchida E, Onda $\mathrm{M}$, et al. Overexpression of acidic and basic fibroblast growth factors in human pancreatic cancer correlates with advanced tumor stage. Cancer Res 1993;53:5289-96.

19. Myers RL, Chded M, Tronick SR, Chiu IM. Different fibroblast growth factor 1 (FGF-1) transcripts in neural tissues, glioblastomas and kidney carcinoma cell lines. Oncogene 1995;1:785-9.

20. Kodama M, Daa T, Kashima K, Yokoyama S, Nakayama I, Noguchi S. Immunohistochemical localization of acidic and basic fibroblast growth factors in human benign and malignant thyroid lesions. Jpn J Clin Oncol 1994;24:66-73.

21. Chao HH, Yang VC, Chen JK. Acidic FGF and EGF are involved in the autocrine growth stimulation of a human nasopharyngeal carcinoma cell line and sub-line cells. Int $\mathrm{J}$ Cancer 1993;54:807-12.

22. Friess H, Yamanaka $\mathrm{Y}$, Buchler $\mathrm{M}$, Beger HG, Do DA, Kobrin MS, et al. Increased expression of acidic and basic fibroblast growth factors in chronic pancreatitis. Am J Pathol 1994;144:117-28.

23. Nabeya Y, Loganzo F, Blundell M, Albino A, Altorki N. Acidic fibroblast growth factor: a possible marker for malignant transformation in Barrett's esophagus (abstract). The Society of Thoracic Surgeons, 1996.

24. Lewin KJ, Appelman HD, Atlas of tumor pathology. Third series, fascicle 18. Tumors of the esophagus and stomach. Washington DC: Armed Forces Institute of Pathology; 1995.

25. Remmele W, Schicketanz K-H. Immunohistochemical determination of estrogen and progesterone receptor content in human breast cancer: computer assisted image analysis (QIC score) vs. subjective grading (IRS). Pathol Res Pract 1993; 189:862-6.

26. Reid BJ, Blount PL, Rubin CE, Levine DS, Haggitt RC, Rabinovitch PS. Flow-cytometric and histologic progression to malignancy in Barrett's esophagus: prospective endoscopic surveillance of a cohort. Gastroenterology 1992:102:1212-9.

27. Younes M, Lebovitz RM, Lechago LV, Lechago J. p53 protein accumulation in Barrett's metaplasia, dysplasia, and carcinoma: a follow-up study. Gastroenterology 1993;105: 1637-42.

28. Reid BJ, Haggitt RC, Rubin CE, Rabinovitch PS. Barrett's esophagus: correlation between flow cytometry and histology in detection of patients at risk for adenocarcinoma. Gastroenterology 1987;93:1-11.

29. Hamelin R, Flejou J, Muzeau F, Potet F, Laurent-Puig P, Fekete $\mathrm{F}$, et al. TP53 gene mutations and p53 protein immunoreactivity in malignant and premalignant Barrett's esophagus. Gastroenterology 1994;107:1012-8.

30. Hardwick RH, Shepherd NA, Moorghen M, Newcomb PV, Alderson D. Adenocarcinoma arising in Barrett's oesophagus: evidence for the participation of p53 dysfunction in the dysplasia/carcinoma sequence. Gut 1994:35:764-8

\section{Discussion}

Dr. Larry R. Kaiser (Philadelphia, Pa.). The study was strictly done with immunohistochemistry, and you have shown previously in evaluating adenocarcinomas that you were able to measure message. Did you in any of these look for message or have you looked at fresh specimens for DNA? I think that the limitations of immunohistochemistry are numerous. It is a qualitative study. It depends on who is grading these specimens. Did a blinded observer look at your immunohistochemistry? How many observers looked at it? The measurements can vary according to the controls. They can vary according to the conditions under which the immunohistochemistry is done. Did you go beyond just the qualitative measurements since you have demonstrated the capability to do it on adenocarcinomas?

Dr. Soslow. To get around the problem of qualitative observation and scoring of the immunohistochemistry, we purposely used this adaptation of the German IRS, which was shown to be equivalent to an image analysis-based quantitation of immunohistochemical reactivity. 